SLAC-PUB-11339

July 2005

\title{
The influence of chain dynamics on the far infrared spectrum of liquid methanol-water mixtures"
}

\author{
K.N. Woods ${ }^{\mathrm{a})}$ \\ BioPhysics Program, Stanford University, Stanford, CA 94309 \\ H. Wiedemann \\ Applied Physics Department, Stanford University, and \\ Stanford Synchrotron Radiation Laboratory, Stanford Linear Accelerator Center, \\ Stanford, CA 94309
}

\begin{abstract}
Far-infrared absorption spectroscopy has been used to study the low frequency $\left(\bullet 100 \mathrm{~cm}^{-1}\right)$ intermolecular modes of methanol in mixtures with water. With the aid of a first principles molecular dynamics simulation on an equivalent system, a detailed understanding about the origin of the low frequency IR modes has been established. The total dipole spectrum from the simulation suggests that the bands appearing in the experimental spectra at approximately $55 \mathrm{~cm}^{-1}$ and $70 \mathrm{~cm}^{-1}$ in methanol and methanol-rich mixtures arise from both fluctuations and torsional motions occurring within the methanol hydrogen-bonded chains. The influence of these modes on both the solvation dynamics and the relaxation mechanisms in the liquid are discussed within the context of recent experimental and theoretical results that have emerged from studies focusing on the short time dynamics in the methanol hydrogen bond network.
\end{abstract}

Submitted to Journal of Chemical Physics

\footnotetext{
* Work supported in part by Department of Energy contract DE-AC02-76SF00515.

a) Electronic mail: knwoods2@lbl.gov
} 


\section{INTRODUCTION}

The underlying microscopic mechanisms contributing to the dynamics in methanol-water mixtures have been explored extensively by numerous experimental and theoretical methods. The interest in the mixture of these two solvents has arisen, not only because of the importance of both molecules as hydrogen-bonding solvents in many chemical applications; but additionally, because it is possible that such a mixture system can also serve as a model for understanding both hydrophobic hydration and solvation in more complex systems such as biomolecules in aqueous environments.

Three distinct relaxation processes have been identified in experimental and molecular dynamics (MD) simulation dielectric relaxation studies on liquid alcohol-water mixtures. ${ }^{1,2,3}$ And the relaxation processes have been ascribed to the following dynamical events taking place in the liquid: a main relaxation process consisting of a cooperative dynamics of the hydrogen-bonded system; a secondary relaxation involving rotation of singly hydrogen bonded molecules at the end of chains; and a third very fast relaxation process originating from the flipping motion of free $\mathrm{OH}$ groups. ${ }^{2}$ Similarly, in MD studies focusing on the solvation dynamics of linear alcohols such as methanol, three stages of solvation have also been identified. ${ }^{4}$ The solvation stages essentially mirror the relaxation processes detected in the dielectric relaxation experiments and include: a Gaussian or inertial relaxation regime at early times, librational motions at intermediate times, and slow, diffusive region at longer times. One question that arises when considering mixtures of polar liquids like methanol and water is: to what extent does the energy lowering effects characterizing the equilibrium solvation process influence the intermolecular dynamics of the liquid system? Although the dynamics of liquids studied by far-infrared (FIR) spectroscopy have generally been described in terms of dielectric properties, recent experimental and theoretical experiments exploring the short-time dynamics of the solvation response have revealed that a comprehensive understanding about specific molecular motions taking place during the solvation process also offers considerable insight into the underlying microscopic processes that contribute to the intermolecular spectrum. ${ }^{5,6,7}$

The aim of the current study is to determine what inferences can be made about the relationship between solvation relaxation and dielectric relaxation in the response function of liquid methanol by exploring the low frequency infrared absorption spectrum of mixtures of water and methanol obtained from a very high brightness source of FIR. ${ }^{8,9}$ With this objective in mind, we will attempt to understand how these two processes (solvation and relaxation) in both molecules dictate the intermolecular dynamics occurring in the mixture system by contrasting our experimental results with those obtained from a first principles MD simulation on a liquid methanol-water mixture in addition to results obtained from numerous other theoretical and experimental studies that have been conducted on equivalent mixture systems. 


\section{EXPERIMENTAL AND COMPUTATIONAL DETAILS A. Experiment}

The methanol used in the experiments was obtained from Sigma-Aldrich. The methanol-water mixtures were prepared by mixing volume fractions of methanol with deionized water; and each mixture solution was consequently filtered before use in an experiment. In this study, the far-infrared spectra of all of the mixtures can be described in terms of their volume fraction of methanol $\left(\mathrm{V}_{\mathrm{ME}}\right)$. The experimental data was collected at the SUNSHINE facility at Stanford University using an intense electron source of farinfrared radiation. ${ }^{9,8}$ In the experiments a dispersive, Fourier Transform technique was used to determine the frequency-dependent absorption coefficients of the samples. ${ }^{10}$ This is accomplished by using a sealed sample cell for the measurements that is composed of a polished silicon window that also serves as a reflector in one arm of the two beam interferometer. The spectral range in the experiments is determined by Fourier Transform of the particle distribution and extends from $5-120 \mathrm{~cm}^{-1}$; although for the experimental analysis, we have only utilized the $10-100 \mathrm{~cm}^{-1}$ region of the spectrum where the intensity of the IR radiation is the strongest.

\section{B. Simulation}

The electronic structure was represented within the Kohn-Sham formulation of the density functional theory (DFT). The gradient-corrected BLYP ${ }^{11,12}$ functional was used in the DFT MD simulation using the Car-Parinello method ${ }^{13}$ and the CPMD code. In the simulation, the electronic states were expanded in a plane wave basis with a cutoff of $70 \mathrm{Ry}$. The fictitious mass associated with the plane wave cutoff was chosen as 900 a.u. and semi-local norm-conserving Martins-Troullier pseudopotentials ${ }^{14}$ were used for the $\mathrm{C}, \mathrm{O}$, and $\mathrm{H}$ atoms to limit the number of electronic states to contain only the valence electrons. The pseudopotential cutoff radii for the atoms used were 1.11, 1.23, and 0.50 a.u. for the $\mathrm{O}, \mathrm{C}$, and $\mathrm{H}$ atoms respectively.

The initial configuration for the mixture containing 16 methanol molecules and 16 water molecules was taken from a $1 \mathrm{~ns}$ MD simulation using the empirical forcefield developed by Haughney et al..$^{15}$ After a subsequent relaxation of the start configuration of approximately $1 \mathrm{ps}$ using the CPMD code, the first principles simulation was

performed on the mixture system in a cubic box with an edge of $11.219 \AA$ for an additional $10 \mathrm{ps}$. A time step of $0.145 \mathrm{fs}$ ( 6.0 a.u. $)$ has been used in the simulation and the temperature in the system held constant at $298 \mathrm{~K}$ by use of a Nosé-Hoover thermostat.

The infrared absorption coefficient is calculated using the dipole correlation function from the simulation and the following equation:

$$
\alpha(v) \cdot n(v)=\frac{4 \pi v \tanh (\beta \hbar v / 2)}{3 \hbar c V} \times \int_{-\infty}^{\infty} d t e^{-i \omega t}\langle\mathrm{M}(t) \cdot \mathrm{M}(0)\rangle,
$$

where $\alpha(v)$ is the frequency dependent absorption coefficient, $n(v)$ is the frequency dependent refractive index, $\beta=1 / \mathrm{k}_{b} \mathrm{~T}$ and $\mathrm{k}_{\mathrm{b}}$ is Boltzmann's constant, $\mathrm{V}$ is the volume, $\mathrm{T}$ is the temperature, $\mathrm{c}$ is the speed of light in vacuum, and $\mathbf{M}$ is the total dipole moment obtained from the last $5 \mathrm{ps}$ of the $10 \mathrm{ps}$ simulation.

Although it is possible that the simulation size ( 32 molecules) may be too small to truly capture the collective properties of dipolar relaxation that are probed in dielectric studies of polar liquids like methanol and water, it is important to note that the far- 
infrared spectrum spans a wavelength region that encompasses both low frequency diffusive motions and a higher frequency resonant region that provides information about the intermolecular interactions that are occurring in the liquid.

From recent experimental and theoretical studies on various hydrogen-bonding liquids, it has been suggested that the rate determining step of the relaxation process is due to the breaking and formation of hydrogen bonds. ${ }^{16,7,5,17}$ Interestingly, simulation results from the work of Luzar et al. on the dynamics of hydrogen bonding in liquid water ${ }^{18}$ have revealed that the collective properties of nearest neighbors have little influence on the fluctuation of the hydrogen bonds in the liquid. Therefore, it is this aspect of the relaxation process that our current far-infrared investigation on liquid methanol and methanol mixtures will focus: the role of intermolecular interactions and fluctuations within the local microscopic structure of the liquid that may influence the underlying mechanism governing relaxation.

\section{RESULTS}

\section{A. Dynamical properties}

As has been shown in a previous first principles MD study on liquid methanol, ${ }^{19}$ the center of mass velocity autocorrelation function (ACF) in the $0-100 \mathrm{~cm}^{-1}$ spectral region features a main diffusion peak close to $25 \mathrm{~cm}^{-1}$ and a secondary shoulder centered at about $70 \mathrm{~cm}^{-1}$. In an analysis of the pure liquid spectrum it has been determined that the shoulder at $70 \mathrm{~cm}^{-1}$ arises from a torsional motion of the hydroxyl atom about the intermolecular hydrogen bond. In this work a, similar MD study has been carried out on a $50 \%$ mixture $\left(\mathrm{V}_{\mathrm{ME}}=0.50\right)$ of liquid methanol and water. In Fig. 1, a comparison of the velocity ACF spectrum of pure liquid methanol with methanol in the $50 \%$ mixture in the same spectral region clearly reveals that changes in the methanol dynamics are occurring with the addition of water.

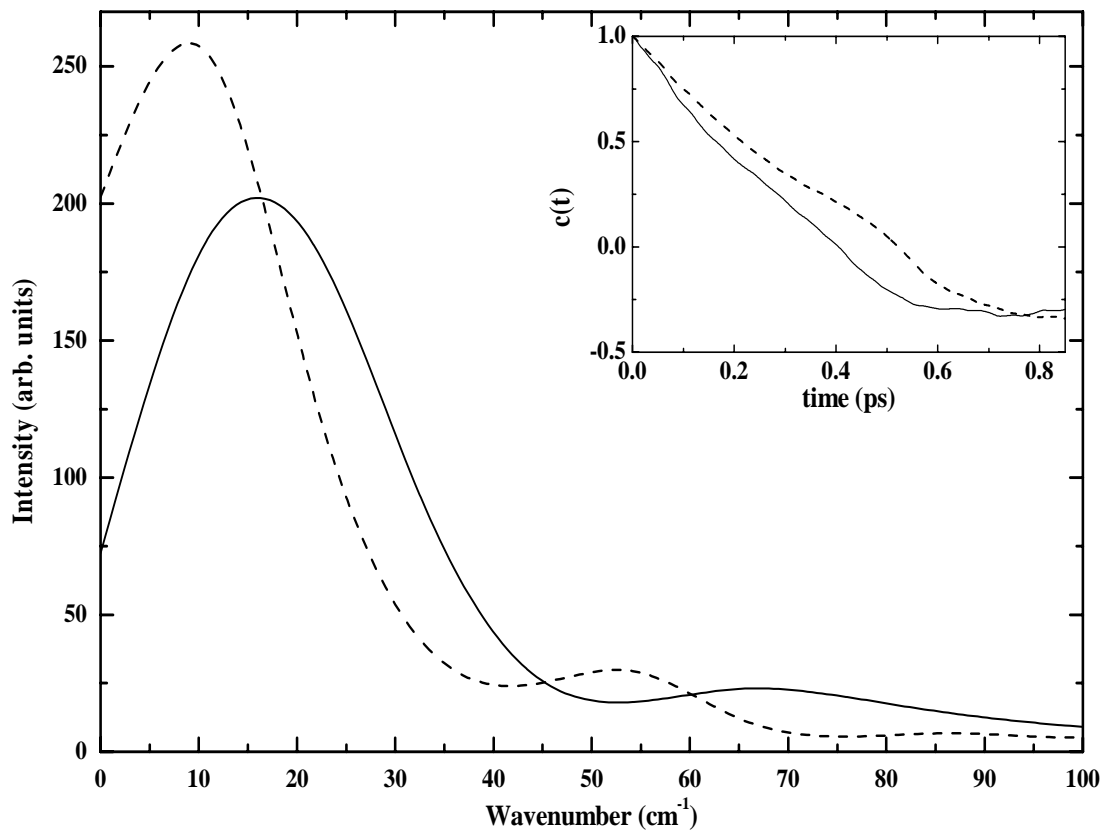

Figure 1: Center of mass velocity autocorrelation function (inset) and power spectrum of pure methanol (solid line) and methanol in a 50\% mixture with water (dashed line). 
A closer inspection into the principal mechanisms contributing to the dynamics in the pure liquid and methanol in the liquid mixture (Figs. 2 and 3) demonstrate the strong influence of the hydroxyl group in the translational dynamics in the former and the increasing importance of motion involving the molecular z-axis in the mixture. More specifically, in Fig. 2 insight into the types of motions contributing to the secondary peak in the velocity ACF spectrum of methanol is gained from a plot considering the contribution of the molecular y-axis and the hydroxyl hydrogen atom in the dynamics. For methanol the molecule lies in the $\mathrm{x}-\mathrm{y}$ plane with the $\mathrm{x}$-axis nearly parallel to the C-O bond.

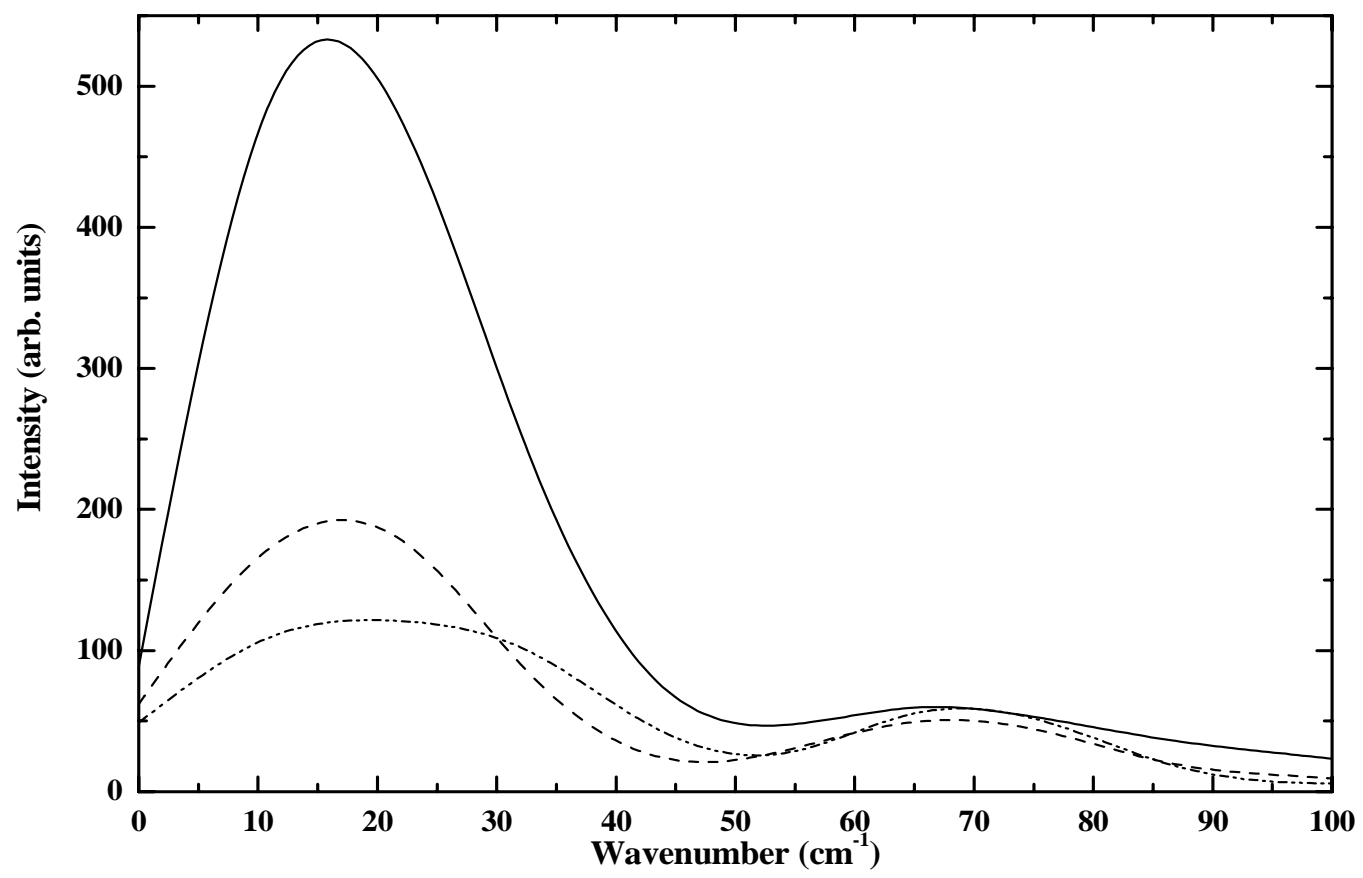

Figure 2: Center of mass velocity autocorrelation function spectrum of methanol and contributions from the methanol hydroxyl hydrogen atom (dash dot-dotted line) and the methanol molecular y-axis (dashed line).

In Fig. 3, a similar analysis is made for the 50\% mixture, where the center of mass spectrum of methanol is contrasted with that derived from the methanol principal axis (zaxis) and also from the methyl hydrogen atoms. With respect to this analysis, it is interesting to note that a peak close in frequency to the shoulder appearing at $55 \mathrm{~cm}^{-1}$ in the center of mass velocity ACF spectrum of methanol in the 50\% mixture (Fig. 1) has also been identified in a crystalline sample of methanol. ${ }^{20}$ The band in the crystalline density of states has been ascribed to methyl group motion, which coincidently, also possesses a strong rotational character. Additionally, a peak at about $85 \mathrm{~cm}^{-1}$ in Fig. 3, that is most apparent in the contribution stemming from the methyl hydrogen atoms, is close in frequency to the reported torsional motion involving the flipping of free hydrogen atoms of isolated water clusters that have been detected in FIR vibrational rotational tunneling experiments. ${ }^{21}$ 


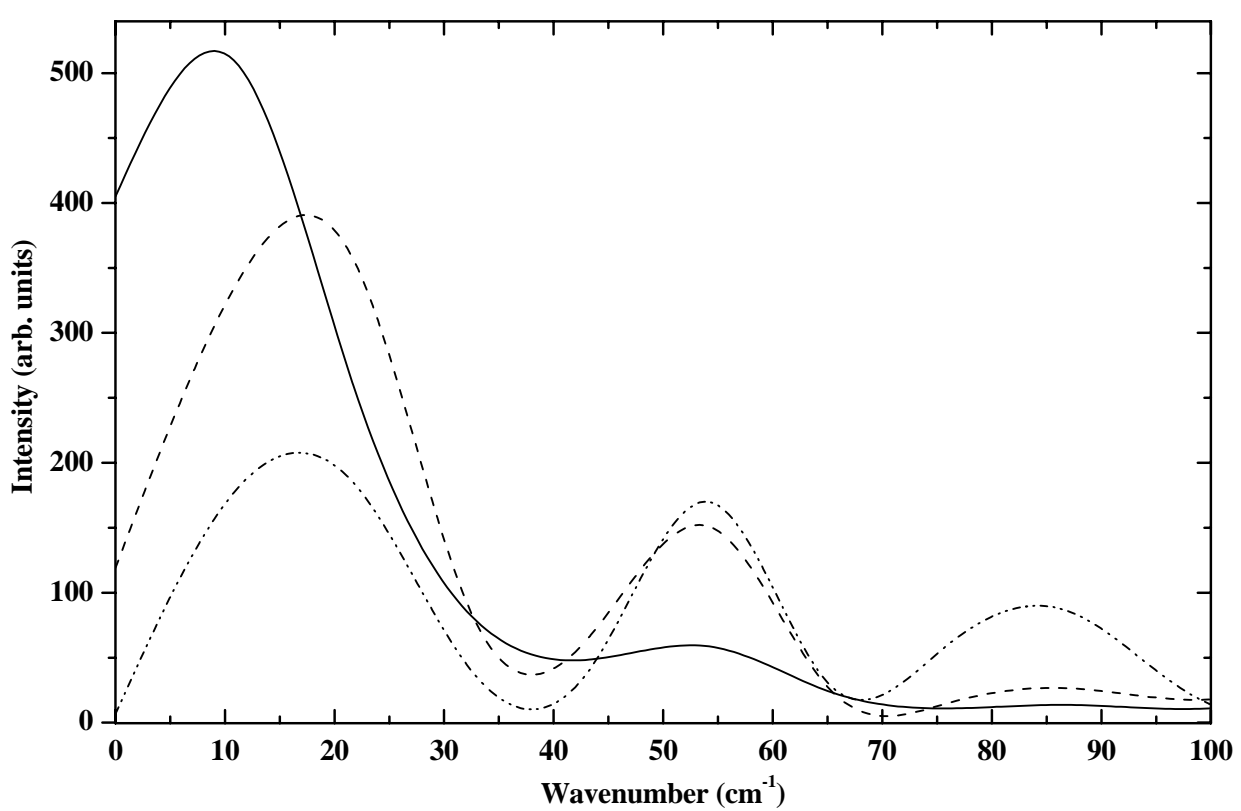

Figure 3: Center of mass velocity autocorrelation function spectrum of methanol in a $50 \%$ mixture with water (solid line) and contributions from the methanol methyl hydrogen atoms (dash dot-dotted line) and the methanol molecular z-axis (dashed line).

\section{B. Hydrogen-bonding}

Hydrogen bond rearrangement is expected to have a strong influence on the intermolecular dynamics of both the pure liquids and the liquid mixtures. Fluctuations within the hydrogen bond network can be characterized by use of a correlation function $(\mathbf{c}(\mathbf{t}))$, which averages over all of the hydrogen-bonded pairs in the liquid,

$$
c(t)=\frac{\left\langle h_{b}(0) \cdot h_{b}(t)\right\rangle}{\left\langle h_{b}{ }^{2}\right\rangle},
$$

and has an existence function of either 0 or $1\left(h_{b}(t)=\{0,1\}\right)$ for a particular hydrogen bond $i$ at time $t$. In this analysis, a methanol molecule is considered to be hydrogenbonded by using a geometrical criterion, where the center of mass distance is less than $3.5 \AA$, the $\mathrm{r}\left(\mathrm{O} \cdots \mathrm{H}_{\mathrm{H}}\right)$ distance is smaller than $2.6 \AA$, and the $\angle \mathrm{HO} \infty \mathrm{O}^{\mathrm{O}}$ angle is smaller than $30^{\circ}$. In Figs. $4 \mathrm{a}$ and $4 \mathrm{~b}$, the hydrogen-bonding correlation spectrum $(\mathbf{c}(\mathrm{v}))$ of pure liquid methanol is contrasted with methanol in the $50 \%$ mixture. Motions within the hydrogenbonding reference frame (inset in Fig. 4a) are divided with respect to those taking place in the direction of the hydrogen bond (A), orthogonal to the bond (B), and motions out of the methanol hydrogen-bonding plane (C).

In comparing the hydrogen-bonding correlations between methanol molecules in the pure and liquid mixture in Fig. 4a and $4 \mathrm{~b}$ respectively, it becomes apparent that the methanol hydrogen-bonding chain network also experiences rather significant changes with the addition of water. The band located at $25 \mathrm{~cm}^{-1}$ in Fig. 4a has been identified as an intermolecular bending mode in previous analyses of hydrogen-bonding correlations in liquid methanol. ${ }^{22,23,24,19}$ And in the pure spectrum (Fig. 4a), the intermolecular bending 
mode is strongly influenced by motion taking place orthogonal to the hydrogen-bonding plane (B-axis). In contrast, the band found at a similar frequency in the mixture spectrum (Fig. 4b), presumably also an intermolecular bending mode, is dominated by motion taking place in the direction of the hydrogen-bonding (A-axis). It is possible that the
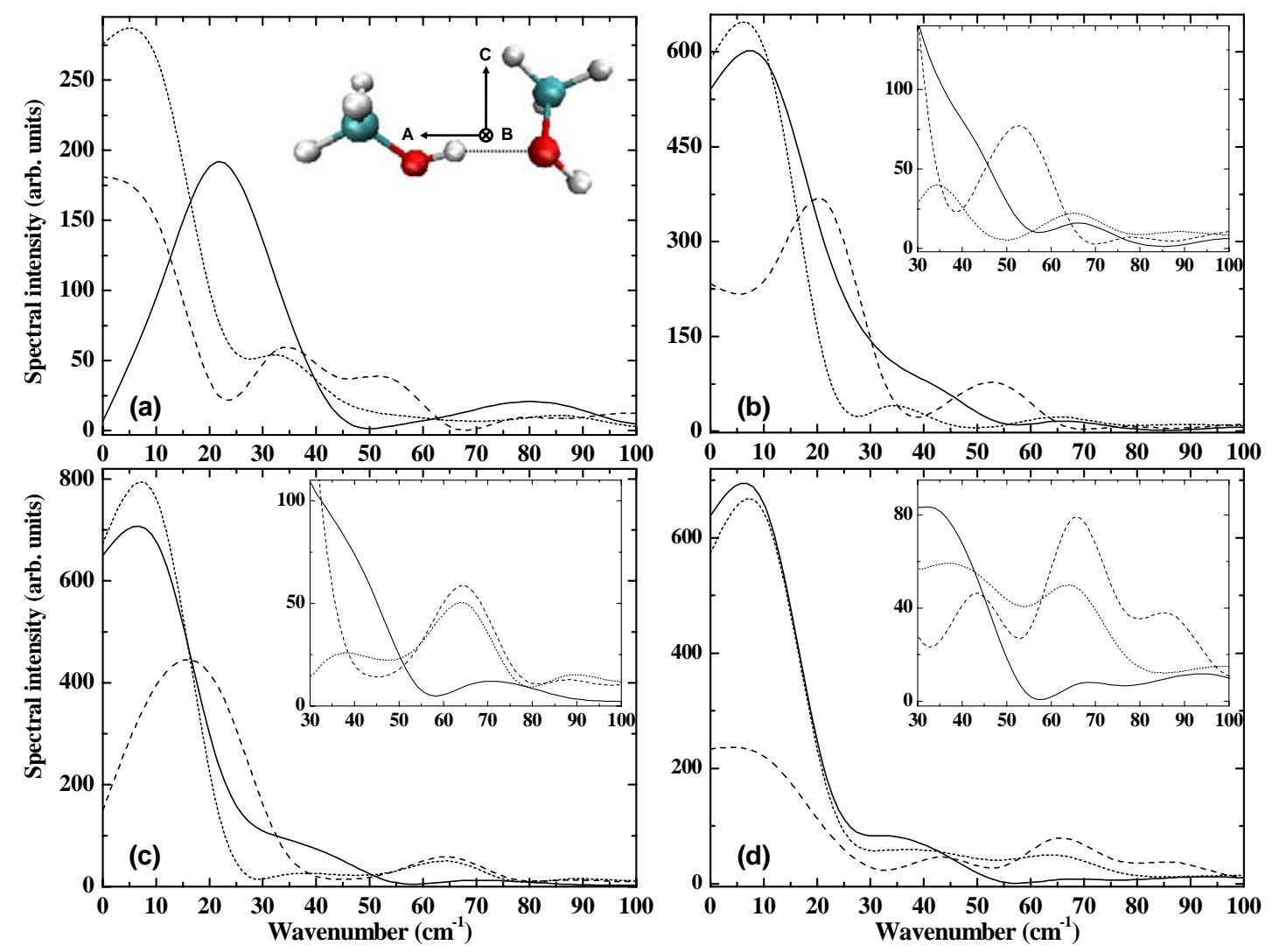

Figure 4: Hydrogen bonding correlation spectrum of:

(a) methanol-methanol associations in pure methanol

(b) methanol-methanol associations in a 50\% methanol-water mixture

(c) methanol-water associations in a 50\% methanol-water mixture

(d) water-water associations in a 50\% methanol-water mixture

calculated using Eq. (2). Motions within the hydrogen-bonding network are defined by use of a reference frame that is shown in the top right corner of Fig. 4a and described in greater detail in section IIIB. The dotted line refers to the A-axis; solid line, the B-axis; and the dashed line, the C-axis. The inset in Figs. $4 \mathrm{~b}-4 \mathrm{~d}$ shows the $30-100 \mathrm{~cm}^{-1}$ region of the correlation spectrum in greater detail.

dissimilarities in the bending modes observed in Figs. $4 \mathrm{a}$ and $4 \mathrm{~b}$ actually reflect a change in the manner in which the methanol molecules diffuse in the chain network of the pure liquid when compared to the liquid mixture. Further analysis of the mode at $25 \mathrm{~cm}^{-1}$ in methanol (Fig. 4a) has revealed that the bending mode is strongly influenced by the methanol oxygen atoms, with only a small contribution from the internal rotation of the methyl group. The intermolecular bending mode in the mixture spectrum (Fig. 4b), which would develop from the correlated motion of methanol molecules hydrogen- 
bonded in the chains, ${ }^{22,23}$ is dominated mainly by methyl group translational motion. Studies on the local structure of methanol in equimolar mixtures with water have revealed that an increasing number of methanol molecules in the chain network acquire three hydrogen bonds ${ }^{25,26,27}$ rather than two, which is the characteristic number for the majority of methanol molecules in the pure liquid. It is possible that a change in the local hydrogen-bonding structure might have an effect on the diffusion process of the interacting methanol molecules.

The $55 \mathrm{~cm}^{-1}$ mode, occurring from motion about the hydrogen-bonding $\mathrm{C}$-axis appears in both the pure and liquid mixture spectrum in Fig. 4a and Fig. 4b respectively. The $55 \mathrm{~cm}^{-1}$ band has previously been identified with methyl group motion in the velocity ACF spectrum in Fig. 1 in section III.A. The intensity of this band is slightly higher in the mixture spectrum than in the methanol spectrum, but due to the overall similarity in both appearance and frequency of the band in both figures, it seems reasonable to assume that an analogous methyl group orientational motion takes place in (methanol) molecules associated in the hydrogen-bonded chain network in the $50 \%$ mixture as in the pure liquid.

Further investigation into correlations arising from motion about the hydrogenbonding B-axis in the $60 \mathrm{~cm}^{-1}$ region of the spectrum, reveals a noticeable red-shift of the peak at $\sim 80 \mathrm{~cm}^{-1}$ in pure liquid spectrum (Fig. 4a) to approximately $65 \mathrm{~cm}^{-1}$ in the mixture spectrum (Fig. 4b). The $80 \mathrm{~cm}^{-1}$ band has been attributed to molecular reorientation about the methanol intermediate axis (y-axis) in an earlier investigations of hydrogen-bonding in liquid methanol. ${ }^{28,29}$ The red-shift of the molecular reorientation peak in Fig. 4b when compared to Fig. 4a suggests a weakening of the hydrogen-bonds in the chain network of the mixture.

From an experimental measurement of the rotational anisotropy decay of the hydroxyl stretch in liquid methanol, it has been determined that excess energy resulting from the excitation is effectively spread throughout the system by an intermolecular energy transfer method ${ }^{30,6}$ that results in faster decay of the excitation. ${ }^{5}$ Subsequent transient hole burning experiments ${ }^{7}$ focusing on the underlying mechanism contributing to the decay process detected in the rotational anisotropy measurements have established that spectral diffusion is responsible for initiating a weakening of the hydrogen bond through a process that involves modulation of the hydrogen bond length, which ultimately facilitates reorientation of the hydroxyl groups of strongly hydrogen-bonded molecules. A measure of the $\mathrm{O}-\mathrm{H}$ bond reorientation of the methanol molecules in the MD simulations carried out in this work is shown in Fig. 5. The O-H bond reorientation is described by use of a reorientational correlation function,

$$
C_{l}(t)=\left\langle P_{l}[\cos \theta(t)]\right\rangle,
$$

where $\mathrm{P}_{l}$ is a Legendre polynomial and $(\mathrm{t})$ is the angle in which the $\mathrm{O}-\mathrm{H}$ bond vector rotates in time $t$. In the figure, there is a noticeable blue-shift of the peak associated with $\mathrm{O}-\mathrm{H}$ reorientation from $\sim 68 \mathrm{~cm}^{-1}$ in the pure liquid to about $75 \mathrm{~cm}^{-1}$ in the mixture spectrum. The apparent shift in frequency suggests a decrease in the orientation rate of the $\mathrm{O}-\mathrm{H}$ bond dynamics in the mixture when compared to pure methanol. Similar results have been obtained from previous MD investigations on the $\mathrm{O}-\mathrm{H}$ reorientation of methanol molecules in liquid methanol -water mixtures. ${ }^{31,26}$ Consequently, the observed shift in the $\mathrm{O}-\mathrm{H}$ bond frequency in Fig. 5 is consistent with the red-shift of the molecular 
orientation band in Fig. 4b, identified in the analysis of hydrogen-bonding correlations taking place between methanol molecules in the mixture.
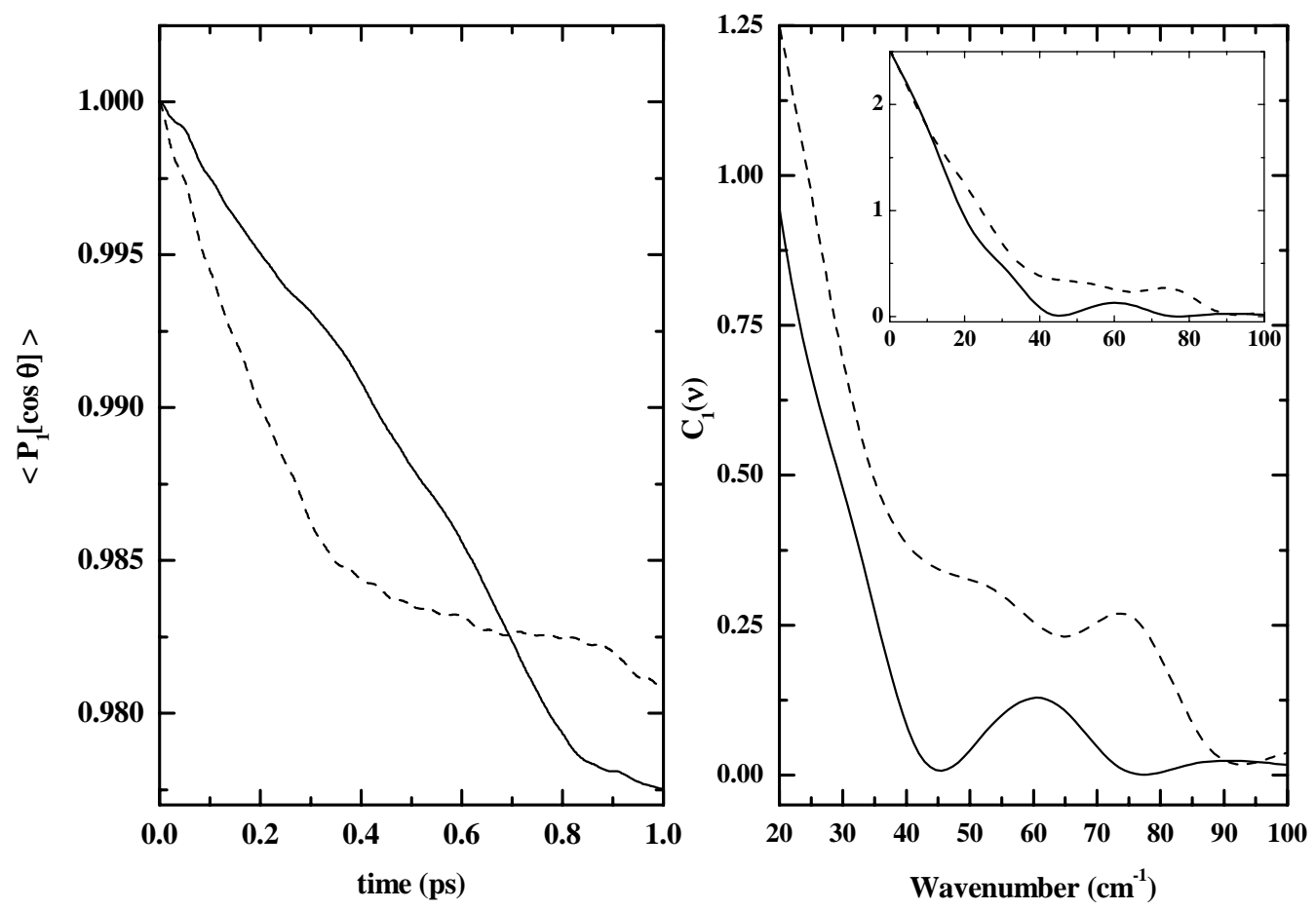

Figure 5: (a) Orientational correlation function of pure methanol (solid line) and methanol in a 50\% mixture with water (dashed line) calculated using Eq. 3. Fourier spectrum of the orientational correlation function of methanol (solid line) and methanol in the mixture (dashed line) in the $20-100 \mathrm{~cm}^{-1}$ spectral region. The inset shows the entire $0-100 \mathrm{~cm}^{-1}$ spectral region.

\section{Molecular flexibility and orientational correlations}

From a three dimensional study on the structural properties of liquid methanol, ${ }^{32}$ it has been suggested that $\mathrm{O}^{\infty} \mathrm{CH}_{3}$ interchain correlations play an important role in the stabilization of the hydrogen-bonded chain network in methanol and also have a strong influence on the translational diffusion properties occurring in the liquid. A subsequent three-dimensional analysis ${ }^{25}$ exploring the local structure of water and methanol in methanol-water mixtures revealed that the competition for hydrogen-bonds in the mixture also plays a role in determining the short-range local order around both molecules and as a consequence, might also have a strong influence on the dynamics occurring in the liquid. MD simulation studies on the dielectric relaxation properties of liquid methanol-water mixtures ${ }^{33,4}$ have determined that the diffusional, but nonexponential decay of the time correlation function (TCF) is both enhanced and accelerated in the transverse component of the dipole density. In the $\bullet 100 \mathrm{~cm}^{-1}$ region of the dielectric relaxation spectrum of reference 1 , a mode at $55 \mathrm{~cm}^{-1}$ has been identified in the transverse portion of the FIR absorption spectrum. Based on results obtained from the three-dimensional study on the local structure of methanol in the alcohol-water 
mixtures ${ }^{25}$ it is conceivable that reorientation of the methyl group will become less hindered due to structural irregularities in the methanol hydrogen bond chain network that develops with increasing amounts of water. Molecular flexibility has been shown to influence the decay rate of excitation in other linear alcohols such as ethanol. In a MD study focusing on the polarizability anisotropy relaxation of liquid ethanol, ${ }^{34}$ torsional motion was found to accelerate the decay rate of both the translational and orientational components of the relaxation. Although ethanol possesses an additional methyl group when compared to methanol, a similar effect has been observed in an earlier analysis of an ab initio MD simulation study on liquid methanol. ${ }^{19}$ In this case, the internal rotational of the methyl group was found to have a close connection with both librational motions and short-range translational motions in the liquid.

In the $100 \mathrm{~cm}^{-1}$ spectral region of the longitudinal portion of the dipole density, a peak in the wave vector-dependent MD analysis ${ }^{1}$ has also been identified at approximately $85 \mathrm{~cm}^{-1}$ in the FIR spectrum. Contrary to the response observed in the transverse portion of the dipole density, the rotational-diffusional part of the longitudinal dipole density was found to gain importance with the addition of water. The increased importance of rotational-diffusion as a relaxation mechanism in the methanol-rich mixtures has been attributed to a less effective canceling of single molecule and collective correlations in the longitudinal response of the dipole density. ${ }^{35}$ Furthermore, a peak identified at about $0.4 \mathrm{ps}$ in the longitudinal dipole density TCF in both methanol ${ }^{1}$ and in the methanol-rich mixtures ${ }^{33}$ was also determined to have a vaguely understood involvement in the relaxation process. Further analysis ${ }^{4}$ revealed that this peak was associated with torsional oscillations in the methanol chain network, which was later surmised to arise from motion of the $\mathrm{C}-\mathrm{O}$ bonds in methanol. In a related MD investigation on the polarizabililty anisotropy relaxation of methanol, ${ }^{36}$ as observed in depolarized Rayleigh scattering (DRS) and optical Kerr effect (OKE) experiments, it was discovered that due to the lack of symmetry of the methanol molecule, relaxation involves a complicated mix of orientational correlations along different molecular axes. In particular, the decay process was found to involve a coupling of $\mathrm{C}-\mathrm{O}$ bond and $\mathrm{O}-\mathrm{H}$ bond dynamics, which consequently lead to faster relaxation of the polarizability anisotropy.

As discussed previously in section I., a secondary relaxation process has been reported in experimental dielectric relaxation studies ${ }^{2}$ on alcohols like methanol, which exist in associating hydrogen-bonded chains. In these studies, the secondary relaxation was interpreted as a rotation of molecules at the end of the chains. Despite the initial identification of the relaxation process with end molecules, subsequent work on the relaxation dynamics of molecular glass forming liquids like methanol ${ }^{37,38,20,39}$ have provided increasing evidence that the secondary relaxation actually arises from a correlation between localized molecular librations and internal molecular motions. The microscopic origin of the relaxation process has been ascribed to the lack of timescale separation between low energy molecular internal motions and phonon modes.

Although it is difficult to speculate whether there is a definite relationship, we have uncovered two low frequency motions in our own simulation study of methanol mixed with water that seemingly exhibit characteristics that are similar to those credited with the secondary relaxation process. These motions include the $\mathrm{O}-\mathrm{H}$ bond torsional motion, initially observed in velocity ACF spectrum of methanol in Fig. 1 and later 
encountered in the analysis of the reorientational motion of the $\mathrm{O}-\mathrm{H}$ bond in the pure and liquid mixture in Fig. 5; and the internal rotation of the methyl group, which has been found to have a significant influence on methanol dynamics in the mixture. The influence of methyl group motion has been most dramatic in the velocity ACF spectrum of methanol in the mixture (Fig. 2) and has also made an appreciable appearance in our investigation of hydrogen-bonding correlations taking place in the liquid (Fig. 4a and 4b).

\section{Translational modes and relaxation}

Results from theoretical and experimental investigations into the dielectric relaxation process in polar liquids, have established that translational modes assist in the relaxation process by accelerating the decay of the orientational correlations in the liquid. $^{40}$ Additionally, translational motions have also been found to reduce the time scale separation between orientational and nonorientational components contributing to dielectric relaxation. ${ }^{34}$ Although not discussed in the wave vector-dependent study of dielectric relaxation in liquid methanol, ${ }^{1}$ further support for the connection between these two contributions to the relaxation process has been gained from an analysis of the cross correlations occurring between the reorientational and collision-induced contributions to the longitudinal dipole density TCF (Fig. 4 in reference 1). The cross correlations produced a small shoulder at about $0.25 \mathrm{ps}$ in the plot of longitudinal component of the dipole density, which corresponds to approximately $130 \mathrm{~cm}^{-1}$ in the frequency spectrum. Coincidently, work investigating the collective hydrogen-bonding correlations in methanol chains using an instantaneous normal mode (INM) analysis, focusing on translational motions in the liquid, ${ }^{22,23}$ has also uncovered bands located around $130 \mathrm{~cm}^{-1}$ in the spectrum. These bands were assigned to collective "stretching" motions within the methanol hydrogen-bonded chains. Additionally, an investigation into the short-time response of solvent relaxation in liquid methanol, explored in the MD simulation study of polarizability anisotropy relaxation ${ }^{36}$ mentioned in the previous section, has established that the initial decay of the orientational component of the polarizability also has a characteristic frequency with a range between $120 \mathrm{~cm}^{-1}-130 \mathrm{~cm}^{-1}$. Although it has been suggested that the short-time and long-time contribution to dielectric relaxation in liquid methanol is mainly rotational in character, ${ }^{1,33}$ it has become increasingly apparent that the intermediate time-scale of the relaxation process ( $0.2-0.6 \mathrm{ps})$ does in fact include an intricate combination of short-range translational and librational motions.

\section{E. Influence of water on methanol dynamics}

Although not mentioned in the discussion regarding hydrogen-bonding correlations between methanol molecules in section III.B., there is a barely noticeable shoulder at about $45 \mathrm{~cm}^{-1}$ in the $50 \%$ methanol-water mixture spectrum in Fig. $4 \mathrm{~b}$ that is noticeably absent from correlations between molecules in the pure spectrum (Fig. 4a). Based on the description of motions with respect to the hydrogen-bonding reference frame, the $45 \mathrm{~cm}^{-1}$ band is mainly associated with motions taking place orthogonal to the intermolecular hydrogen bond. Additionally, we have found that this peak is strongly manifested in the velocity ACF spectrum of the hydroxyl hydrogen atom (Fig. 6) in the mixture system. Interestingly, a band at a similar frequency also appears in both the methanol-water and water-water hydrogen bonding correlation spectra in the simulation 
mixture in Fig. 4c and Fig. 4d respectively. It is possible that the change in the translational characteristics of the methanol hydroxyl atom in the mixture, when compared with the pure liquid reflects the growing influence of water to the methanol dynamics.

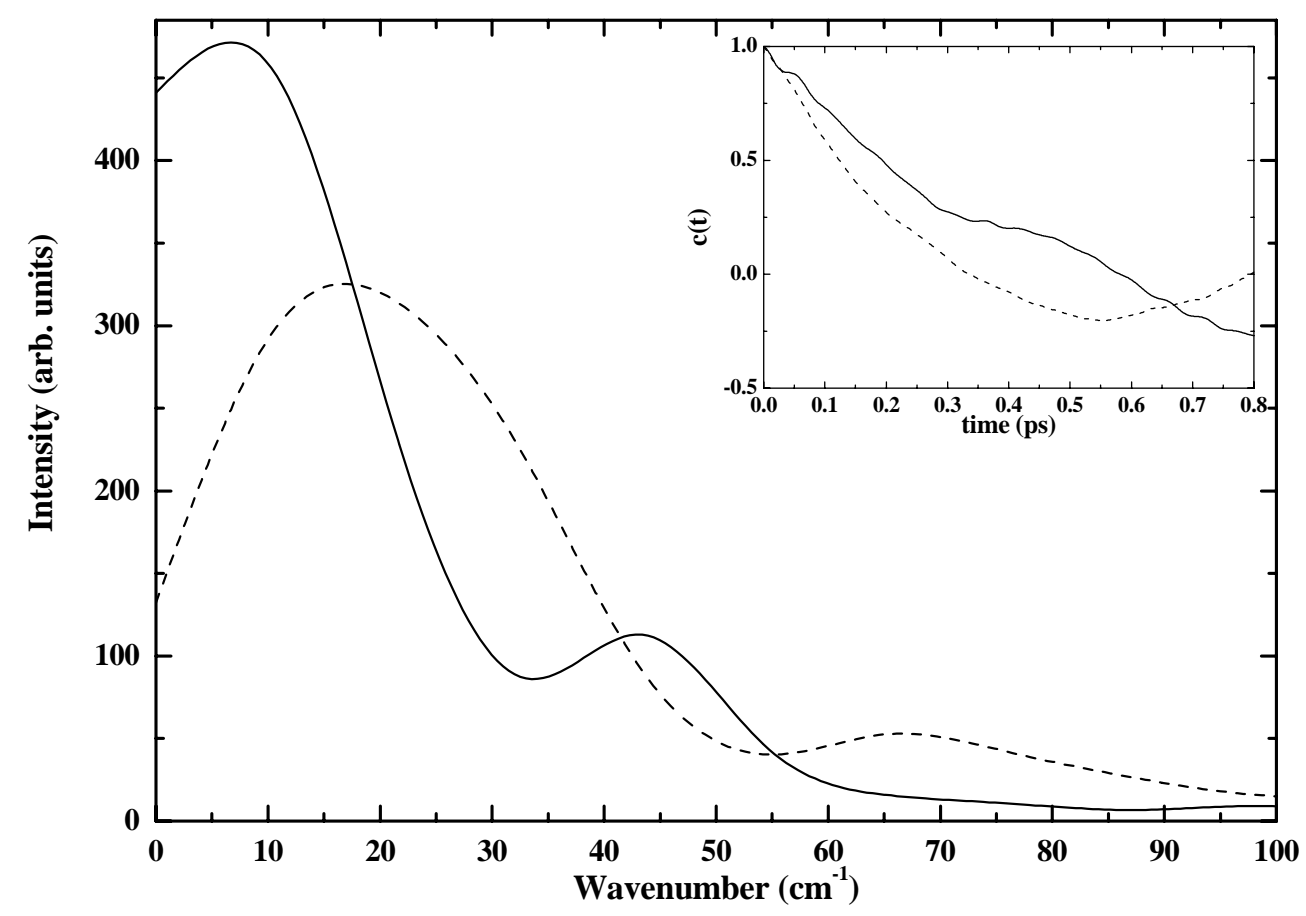

Figure 6: Velocity autocorrelation function (inset) and power spectrum of the methanol hydroxyl hydrogen atom in pure methanol (dashed line) and methanol in the 50\% mixture with water (solid line).

It should also be noted that the hydroxyl atom correlations in the time domain (inset of Fig. 6) also produce slight peaks at approximately $55 \mathrm{fs}$ and $450 \mathrm{fs}$ in the mixture that are apparently absent in pure methanol. The small bulge at $450 \mathrm{fs}$ is also present in the center of mass velocity ACF of methanol in the mixture (inset of Fig 1). The peaks found in the correlation function in Fig. 6 correspond to approximately 600 $\mathrm{cm}^{-1}$ and $75 \mathrm{~cm}^{-1}$ in the frequency domain. IR pump-probe spectroscopy experiments probing the hydrogen-bonding dynamics in liquid methanol $1^{41,42}$ have determined that hydrogen bond dissociation occurs on two different timescales. For a population of the molecules, direct hydrogen bond breaking occurs on a very fast time-scale and for another, an indirect method of hydrogen bond dissociation results in a slower time constant. Curiously, the time-scale of both mechanisms ${ }^{43}$ coincides with the peaks observed in the velocity ACF of the methanol hydroxyl group in the mixture with water (inset of Fig. 6).

An earlier velocity correlation function analysis on correlated motions in liquid water has determined that the diffusion process involves translational motion as well as a rigid rotation of molecules interacting through hydrogen bonds. ${ }^{44}$ In particular, the 50 $\mathrm{cm}^{-1}$ mode, which has been described as an intermolecular bending vibration in liquid water, ${ }^{45}$ was found to possess an additional rotational component. Based on the results 
from the velocity projections on water, ${ }^{44}$ the rotational component of the bending mode consists of a correlated librational motion of hydrogen-bonded molecules about the water molecule's smallest moment of inertia. Perhaps then, it is no coincidence that the band at $\sim 45 \mathrm{~cm}^{-1}$ in the velocity ACF spectrum of methanol in the mixture (Fig. 5) develops from the coupling of the internal rotation of the methyl group to the molecular librational motion of methanol about its inertial $x$-axis. This is in sharp contrast to the diffusional motion of the hydroxyl atom in the pure liquid spectrum, where the dynamics appear to be influenced mostly by motion in the direction of the hydrogen bond. The dissimilarity of the hydroxyl atom motion in the two liquids is in agreement with the results obtained from the analysis of the velocity ACF spectrum in Fig. 1 and Fig. 2 in section III.A. In this case, the hydroxyl atom appears to dominate the center of mass velocity ACF of pure methanol; while in the mixture, we find that the translational dynamics of methanol is more influenced by methyl group motion.

Also neglected in section III.B. was reference to the almost unnoticeable increase of the C-axis contribution (motion out of the hydrogen-bonding plane) to the $65 \mathrm{~cm}^{-1}$ mode in the methanol hydrogen-bonding correlation spectrum in Fig. $4 \mathrm{~b}$. In fact, the 65 $\mathrm{cm}^{-1}$ mode in Fig. 4b was only considered in the context of molecular reorientation. Although it is clear that the peak in the mixture spectrum has undergone a small, frequency shift, it is possible that changes to the peak, with respect to contributions associated with hydrogen-bonding reference frame, may indicate a more significant transformation occurring in the network. A previous investigation into the hydrogenbonding interactions in pure liquid methano ${ }^{19}$ has uncovered that correlations involving motion out of the hydrogen-bonding plane (C-axis) appear as modes in the $>100 \mathrm{~cm}^{-1}$ region of the spectrum. Specifically, in the low frequency spectral region $\left(0-200 \mathrm{~cm}^{-1}\right)$, the bands associated with $\mathrm{C}$-axis motion in the pure liquid were believed to originate from a complex mixture of librational and correlated stretching motions taking place in the hydrogen bond network, which consequently resulted in peaks at approximately 130 $\mathrm{cm}^{-1}$ and $150 \mathrm{~cm}^{-1}$ in the spectrum.

Returning to the discussion regarding the $65 \mathrm{~cm}^{-1}$ mode in Fig. $4 \mathrm{~b}$, it is worth noting that cross-correlations between methanol and water molecules interacting through hydrogen bonds in the mixture (Fig. 4c) also produce a peak at $65 \mathrm{~cm}^{-1}$ that is apparently influenced by motion involving the hydrogen-bonding $\mathrm{C}$-axis. It is highly likely that the $65 \mathrm{~cm}^{-1}$ mode in the methanol hydrogen-bonding spectrum (Fig. 4b) is yet another indication of water's influence on methanol dynamics, and quite possibly suggests a change in the local structure of a fraction of the methanol molecules in the mixture.

Additionally, a $65 \mathrm{~cm}^{-1}$ peak is also present in the plot of water hydrogen-bonding autocorrelations in the mixture (Fig. 4d). Based on the similarity of the peak locations in the hydrogen-bonding spectrum in Fig. 4d to those in the experimental FIR absorption spectrum of pure liquid water, ${ }^{10}$ it is possible that the $65 \mathrm{~cm}^{-1}$ mode in Fig. $4 \mathrm{~b}$ and possibly Fig. $4 \mathrm{c}$ have a similar origin. The $65 \mathrm{~cm}^{-1}$ band in the FIR absorption of pure liquid water has been attributed to a bending mode of water molecules arranged in a configuration that is less structured than would be encountered in either ice or the supercooled liquid. ${ }^{46}$ 


\section{F. FIR experimental and simulation spectra \\ 1. Methanol-rich}

The experimental FIR absorption spectrum of liquid methanol-water mixtures is shown in Fig. 7, where the spectrum of pure liquid water and methanol are displayed in Fig. 7a and Fig. 7e respectively. In the FIR spectrum of methanol, there is a prominent peak found at about $70 \mathrm{~cm}^{-1}$ that has previously been linked with the torsional motion of the hydroxyl group. In the methanol-rich mixture $\left(\mathrm{V}_{\mathrm{ME}}=0.75\right)$ in Fig. $7 \mathrm{~d}$, the peak found at $70 \mathrm{~cm}^{-1}$ in the pure liquid is slightly blue-shifted and peaks at about $75 \mathrm{~cm}^{-1}$ in the methanol-rich mixture spectrum. Although the presence of the water dynamics cannot be entirely neglected in the methanol-rich mixture, it is conceivable that the dominant polar liquid in the mixture will exhibit stronger FIR bands than its solute counterpart.

Therefore, in the analysis of the methanol-rich FIR absorption spectrum we will focus mainly on how the bands in the spectrum relate to methanol structure and dynamics.

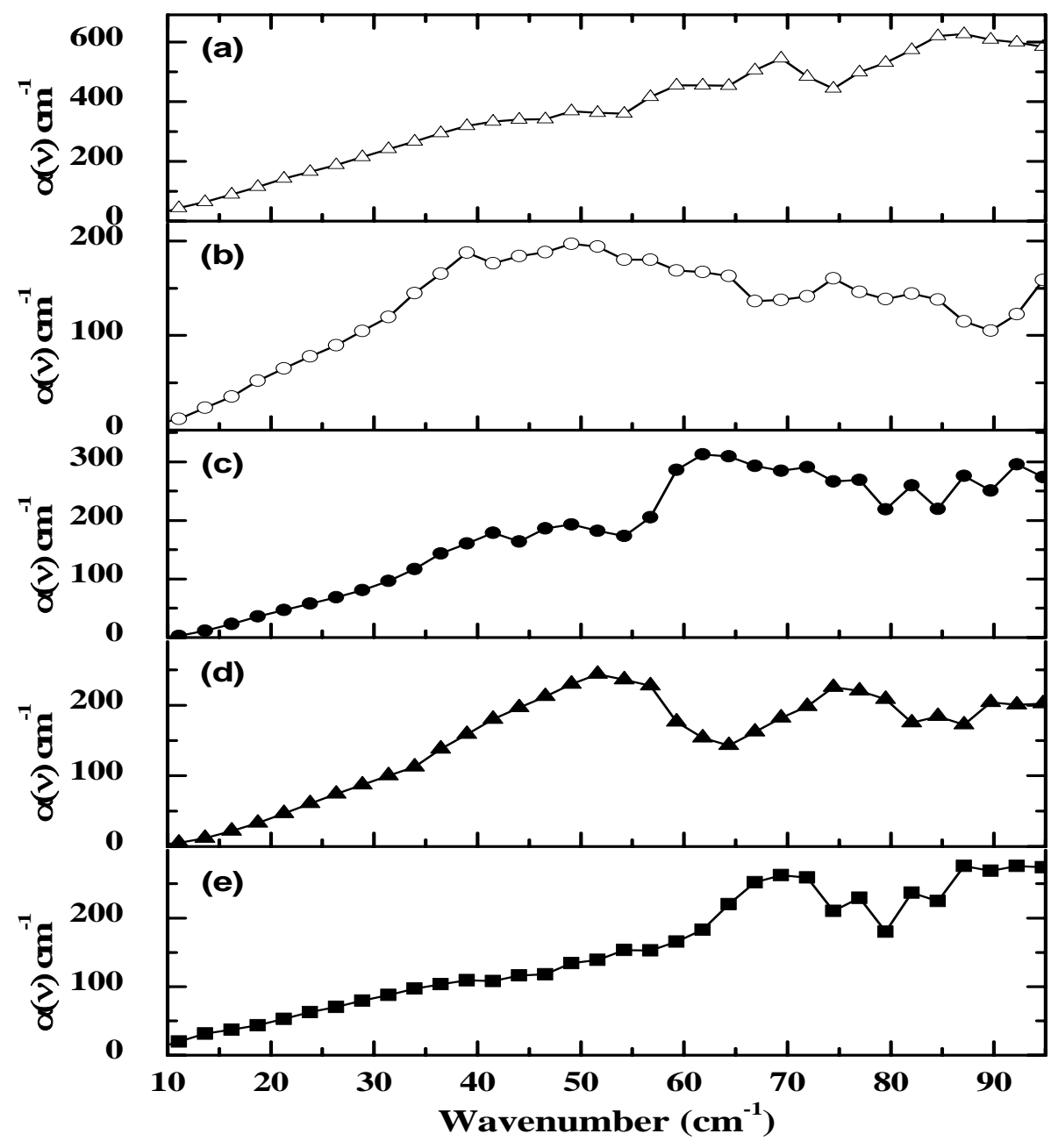


Figure 7: Experimental FIR absorption spectrum of
(a) pure water
(b) $\mathrm{V}_{\mathrm{ME}}=0.25$
(c) $\mathrm{V}_{\mathrm{ME}}=0.50$
(d) $\mathrm{V}_{\mathrm{ME}}=0.75$
(e) pure methanol

at $298 \mathrm{~K}$ in the $10-95 \mathrm{~cm}^{-1}$ spectral region at a $2 \mathrm{~cm}^{-1}$ resolution.

Based on the trend of the O-H bond dynamics in Fig. 5, the shift of the $70 \mathrm{~cm}^{-1}$ mode is believed to be attributed to methanol O-H orientational motion. In Fig. 7d, a peak close to $50 \mathrm{~cm}^{-1}$ is also observed in the spectrum that did not appear in the pure methanol IR absorption spectrum (Fig. 7e). A band in the same frequency region has also been identified in the center of mass velocity ACF spectrum in Figs. 1 and 2, in addition to the hydrogen-bonding correlation spectra of methanol in the pure and $50 \%$ mixture (Figs. 4a and 4b). The results from several different MD analyses of liquid methanol-water mixtures ${ }^{27,25,33}$ have determined that the addition of small amounts of water to the mixture, leads to water molecule aggregation rather than a preference for interaction with the methanol network. In a MD analysis of the structural arrangement of methanol molecules in the methanol-rich mixtures, ${ }^{25}$ it has been revealed that the local structure within the methanol chain network changes very little when small amounts of water are added; while interactions between chains become less regular. We have speculated in this paper that the change in interchain interactions in the methanol network may account for the increased orientational motion of the methyl group. Furthermore, we have labeled the band located at $55 \mathrm{~cm}^{-1}$ in the $<100 \mathrm{~cm}^{-1}$ region of the velocity ACF (Fig. 2) and also in the hydrogen-bonding correlation spectrum of methanol (Figs. 4a and $4 \mathrm{~b})$ with this methyl group orientational motion. It is possible that this band is conspicuously absent from Fig. 7e simply because the contribution is too small to be observed in the pure methanol FIR absorption spectrum.

\section{50\% mixture}

In the $\mathrm{V}_{\mathrm{ME}}=0.50$ mixture (Fig. $7 \mathrm{c}$ ) there is a very broad band in the spectrum that extends from approximately $50 \mathrm{~cm}^{-1}$ to $75 \mathrm{~cm}^{-1}$. Based on the complexity of correlations occurring in the mixture system, it is difficult to hypothesize about the origin of this very broad band in the FIR spectrum without some guidance from the simulation results on a comparable system. Although an oversimplification in terms of a description of the mixture system, in Fig. 8 the simulation total dipole absorption spectrum is divided into components relating to the principal axes of the two molecules making up the mixture (methanol and water), with the expectation that it may provide some insight into the origin of the peaks.

Based on a comparison with the CPMD IR absorption spectrum in Fig. 8, it is likely that the broad band centered at about $70 \mathrm{~cm}^{-1}$ in the experimental absorption spectrum (Fig. 7c) is actually composed of two separate bands: a peak centered at about $60 \mathrm{~cm}^{-1}$ and another at around $75 \mathrm{~cm}^{-1}$. A peak close to $60 \mathrm{~cm}^{-1}$ has already been identified with a band that has appeared in experimental FIR ${ }^{10}$ and Raman studies ${ }^{46}$ on liquid water (in section III.E.); and from these studies has been attributed to a bending mode of water molecules interacting in a less structured local configuration. The $75 \mathrm{~cm}^{-1}$ experimental mode (Fig. 7c) appears at a slightly lower frequency $\left(\sim 5 \mathrm{~cm}^{-1}\right)$ than the band found around the same frequency in the simulation spectrum (Fig. 8). Based on the evolution of a similar band found in both methanol and in the methanol-rich mixture 
absorption spectrum in Fig. 7e and Fig. 7d respectively, it is highly likely that this band is associated with $\mathrm{O}-\mathrm{H}$ bond reorientation.

Although there is very low intensity in the $<50 \mathrm{~cm}^{-1}$ region of the CPMD spectrum, ${ }^{47}$ there is a discernable peak arising from the molecular $\mathrm{z}$-axis at $55 \mathrm{~cm}^{-1}$ and another peak centered at approximately $45 \mathrm{~cm}^{-1}$ that possess contributions from both the molecular $\mathrm{x}$ - and $\mathrm{z}$-axes. The $55 \mathrm{~cm}^{-1}$ mode has been accredited with motion of the methanol methyl group numerous times within this text. The small band at approximately $45 \mathrm{~cm}^{-1}$ in Fig. 8 has been implicated with dynamics of the methanol hydroxyl atom in the mixture system (Fig 6); and additionally with hydrogen bond autoand cross-correlations of water and methanol molecules in the mixture (Figs. 4b-4d). Since a peak at approximately $40 \mathrm{~cm}^{-1}$ has also been described as an intermolecular bending mode in both experimental and theoretical studies on supercooled water and ice, ${ }^{48,49}$ it is possible that the $\mathrm{x}$-axis contribution to the peak at $45 \mathrm{~cm}^{-1}$ in Fig. 8 characterizes the water bending mode uncovered in those studies. Furthermore, in the $50 \%$ mixture a population of the methanol molecules is expected to contain three hydrogen-bonds (two acceptor and one donor hydrogen-bond). MD simulation studies exploring the local ordering of methanol molecules equally mixed with water, ${ }^{25}$ suggest that methanol adopts a tetrahedral ordering in the mixture that somewhat resembles water in an "ice-like" configuration. ${ }^{25}$ Based on the presence of the $45 \mathrm{~cm}^{-1}$ mode in the velocity ACF spectrum of the methanol hydroxyl atom in the mixture (Fig. 6), the z-axis contribution at $45 \mathrm{~cm}^{-1}$ in Fig. 8 is believed to originate from motion resulting from the mixed internal rotation/librational mode of methanol molecules that are influenced by the dynamics of water.

Although discussion pertaining to the simulation spectrum has mainly been restricted to the spectral region that overlaps with the experimental spectral range, it is also interesting to consider intermolecular vibrations occurring in the higher frequency region, even if we are unable to explore them experimentally. In Figs. 9a and 9b, the low frequency simulation IR absorption spectrum in the $100-200 \mathrm{~cm}^{-1}$ region is shown. Comparison of the pure and mixture spectrum reveals that $130 \mathrm{~cm}^{-1}$ mode, which has been associated with both methyl group internal rotation ${ }^{50}$ and correlated stretching modes, ${ }^{51,22,23}$ increases from a shoulder in the pure spectrum to a prominent peak at about $120 \mathrm{~cm}^{-1}$ in the mixture spectrum. Also observed, is a noticeable peak located at $170 \mathrm{~cm}^{-1}$ that is not as clearly formed in the pure methanol absorption spectrum in Fig. 9a. A peak close to $170 \mathrm{~cm}^{-1}$ has previously been described as an intermolecular stretching mode in experimental studies on liquid water. ${ }^{52,53,45}$ In the higher frequency spectrum (inset in both Figs. 9a and 9b), peaks that have been identified with librational motions about the smallest inertial axes in methanol ${ }^{4,27,54,55,22,55,56,57}$ and water ${ }^{58,44}$ peak close to $600 \mathrm{~cm}^{-1}$. Additionally, the peak at about $400 \mathrm{~cm}^{-1}$, which is slightly more prominent in the mixture spectrum, has been accredited with the librational motion about the molecular x-axis of hydroxyl hydrogen atoms at the end of chains in pure liquid methanol that are not participating in hydrogen bonds. ${ }^{50}$

\section{Water-rich}

The water-rich experimental absorption spectrum $\left(\mathrm{V}_{\mathrm{ME}}=0.25\right)$ in Fig. $7 \mathrm{~b}$ features an extremely broad band centered at $\sim 50 \mathrm{~cm}^{-1}$ and another band centered at approximately $75 \mathrm{~cm}^{-1}$. Simulation results on an equivalent methanol-water mixture 
suggest that methanol molecules are completely infused into the water hydrogen bond network in water-rich solutions, and as a consequence only interact with other methanol molecules through secondary correlations. ${ }^{25}$ It is also at this concentration $(X<0.3)$, that the secondary relaxation processes ceases to be detected in the experimental dielectric studies of chain forming alcohol-water mixtures, ${ }^{2}$ providing some support that this relaxation may depend on specific interactions between methanol molecules arranged in chains. On the other hand, water molecules in water-rich solutions have been found to adopt an almost ideal tetrahedral local arrangement in the presence of small amounts of methanol. ${ }^{25}$ If one reconsiders the evolution of the peaks in Fig. 7, it becomes more apparent that the extremely broad band centered at $50 \mathrm{~cm}^{-1}$ in Fig. $7 \mathrm{~b}$ may originate from the $40 \mathrm{~cm}^{-1}$ shoulder in pure water. As has been previously mentioned, a broad peak between $40-50 \mathrm{~cm}^{-1}$ has been described as a bending mode of water molecules interacting in a more structured local arrangement. The strong presence of this bending mode in the spectrum may indicate that a larger fraction of molecules are involved in this hyper-structured configuration than would normally be found in the pure room temperature liquid. The band centered at a slightly higher frequency $\left(75 \mathrm{~cm}^{-1}\right)$ in Fig. $7 \mathrm{~b}$ bears some resemblance to the $65 \mathrm{~cm}^{-1}$ peak in the pure liquid water spectrum. In fact, this mode (at $65 \mathrm{~cm}^{-1}$ ) in liquid water has already been labeled as a secondary bending mode in section III.E., arising from interactions of water molecules in a less structured arrangement. The shift if frequency from $65 \mathrm{~cm}^{-1}$ to approximately $75 \mathrm{~cm}^{-1}$ might imply a strengthening of the hydrogen bond between the molecules participating in this particular structural coordination.

\section{CONCLUSIONS}

Far-infrared absorption spectroscopy has been used to explore the intermolecular associations in liquid methanol-water mixtures. We have uncovered bands in the low frequency spectra that may help provide further insight into the relaxation mechanisms of methanol in the pure and liquid mixtures. Using an $a b$ initio MD simulation of a liquid methanol-water mixture to aid in the interpretation of the experimental data, it has been determined that both the translational and rotational characteristics of methanol change with the addition of water.

The experimental results on the pure and liquid mixtures of methanol and water are in good agreement with the total dipole spectra calculated from the MD simulations carried out in this study. From the simulation of the methanol-water mixture, an analysis of the velocity and hydrogen-bonding correlations of methanol in the liquid mixture $\left(\mathrm{V}_{\mathrm{ME}}=0.50\right)$ has revealed that motion involving the methanol methyl groups is accentuated when water is added; and a concurrent weakening of the hydrogen bonds in the hydrogen-bonding network of methanol is observed. Additionally, there is an observed decrease in the orientational rate of the methanol $\mathrm{O}-\mathrm{H}$ bond dynamics in the liquid mixture. Fundamental changes detected in the methanol hydroxyl hydrogen atom dynamics in the simulated $50 \%$ methanol-water mixture may also indicate a strong influence of water-like dynamics on the types of methanol-methanol interactions taking place in the liquid mixture.

Recent experimental investigations into the solvation dynamics in methanol have uncovered both translational and rotational motions important in the relaxation pathway of the energy decay. ${ }^{6,5,7}$ The intra- and intermolecular modes identified during the 
intermediate timescale of the decay process seem to parallel those identified in our experimental and simulation studies on the low frequency dynamics in liquid methanol. Specifically, these modes include a torsional motion of the methanol O-H bond and the reorientational motion of the methyl group, which appear as bands in the absorption spectra of methanol and the methanol-rich mixture at about $70 \mathrm{~cm}^{-1}$ and $55 \mathrm{~cm}^{-1}$ respectively. It is probably no coincidence that these modes also appear to simultaneously describe the secondary relaxation process or $\beta$-relaxation that has been resolved in simulation studies of various chain forming liquids near the glass transition temperature $\left(\mathrm{T}_{\mathrm{g}}\right)$. The underlying mechanism for the secondary relaxation has been attributed to a local rearrangement taking place within a minima of the potential energy, involving an intramolecular reorientation coupled to a restricted librational motion. For instance, in ethanol the relaxation process is believed to arise from an internal rotation of the molecular C-O bond strongly coupled to a localized libration of the O-H bond. ${ }^{37}$ Earlier in section III.C. it was hypothesized that the addition of water to methanol might enhance this type of internal torsional motion of the methyl group in liquid methanol.

Although far from a complete description of the intermolecular dynamics taking place in the liquid, it is our hope that the current study on liquid methanol-water mixtures will provide additional insight into the often overlooked low frequency mechanisms that may contribute to the solvation and relaxation dynamics in associating liquids like methanol and water.

\section{ACKNOWLEDGMENTS}

This work was funded by the US Department of Energy, Basic Energy Science Contract No. DE-AC03-76SF00515. We are also grateful for the computational time provided by the Stanford Center for Biomedical Computation.

\section{REFERENCES}

M. S. Skaf, T. Fonseca, and B. M. Ladanyi, J. Chem. Phys. 98 (11), 8929 (1993). T. Sato and R. Buchner, J. Chem. Phys. 118, 4606 (2003).

J. T. Kindt and C. A. Schmuttenmaer, J. Phys. Chem. 100, 10373 (1996). M. S. Skaf and B. M. Ladanyi, J. Phys. Chem. 100, 18258 (1996).

K. J. Gaffney, I. R. Piletic, and M. D. Fayer, J. Chem. Phys. 118, 2270 (2003).

L. K. Iwaki and D. D. Dlott, J. Phys. Chem. A 104, 9101 (2000).

I. R. Piletic, K. J. Gaffney, and M. D. Fayer, J. Chem. Phys. 119, 423 (2003).

P. Kung, H. Lihn, and H. Wiedemann, Phys. Rev. Lett. 73, 967 (1994).

H. Lihn, D. Bocek, C. Settakorn, and H. Wiedemann, Phys. Rev. E 53, 6413 (1996).

K. N. Woods and H. Wiedemann, Chem. Phys. Lett. 393, 159 (2004).

A. D. Becke, Phys. Rev. A 38, 3098 (1988).

C. Lee, W. Yang, and R. C. Parr, Phys. Rev. B 37, 785 (1988).

R. Car and M. Parrinello, Phys. Rev. Lett. 55, 2471 (1985).

N. Troullier and J. L. Martins, Phys. Rev. B 43, 1993 (1991).

M. Haughney, M. Ferrario, and I. R. McDonald, J. Phys. Chem. 91, 4934 (1987).

H.-K. Nienhuys, R. A. van Santen, and H. J. Bakker, J. Chem. Phys. 112 (19), 8487 (2000). 
C. P. Lawrence and J. L. Skinner, Chem. Phys. Lett. 369, 472 (2003).

A. Luzar and D. Chandler, Phys. Rev. Lett. 76 (6), 928 (1996).

K. N. Woods and H. Wiedemann, submitted to J. Chem. Phys (2005).

F. J. Bermejo, J. Alonso, A. Criado, F. J. Mompeán, and J. L. Martínez, Phys. Rev. B 46, 6173 (1992).

F. N. Keutsch and R. J. Saykally, Proc. Natl. Acad. Sci. USA 98, 10533 (2001).

G. Garberoglio and R. Vallauri, J. Chem. Phys. 115, 395 (2001).

G. Garberoglio, R. Vallauri, and G. Sutmann, J. Chem. Phys. 117, 3278 (2002).

J. Marti, J. A. Padró, and E. Guárdia, J. Molecular Liquids 64, 1 (1995).

A. Laaksonen, P. G. Kusalik, and I. M. Svishchev, J. Phys. Chem A 101, 5910 (1997).

M. Ferrari, M. Haughney, I. R. McDonald, and M. L. Klein, J. Chem. Phys. 93

(7), 5156 (1990).

D. S. Venables and C. A. Schmuttenmaer, J. Chem. Phys. 113, 11222 (2000).

M. Matsumoto and K. E. Gubbins, J. Chem. Phys. 93 (3), 1981 (1990).

D. S. Venables and C. A. Schmuttenmaer, J. Chem. Phys. 113, 3249 (2000).

T. Förster, Ann. Phys. 6, 55 (1948).

E. J. Wensink, A. C. Hoffmann, P. J. van Maaren, and D. van der Spoel, J. Chem. Phys. 119, 7308 (2003).

I. M. Svishchev and P. G. Kusalik, J. Chem. Phys. 100 (7), 5165 (1994).

B. M. Ladanyi and M. Skaf, J. Phys. Chem. 100, 1368 (1996).

M. Paolantoni and B. M. Ladanyi, J. Chem. Phys. 117 (3856) (2002).

D. Kivelson and H. Friedman, J. Phys. Chem 93, 7026 (1989).

B. M. Ladanyi and Y. Q. Liang, J. Chem. Phys. 103 (15), 6325 (1995).

M. A. González, E. Encisco, F. J. Bermejo, M. Jiménez-Ruiz, and M. Bée, Phys. Rev. E 61, 3884 (2000).

F. J. Bermejo, W. S. Howells, M. Jiménez-Ruiz, M. A. González, D.L. Price, M.

L. Saboungi, and C. Cabrillo, Phys. Rev. B 69, 174201 (2004).

C. Hansen, F. Stickel, T. Berger, R. Richert, and E. W. Fischer, J. Chem. Phys. 107 (4), 1086 (1997).

B. Bagchi and A. Chandra, Phys. Rev. Lett. 64, 455 (1990).

J. B. Asbury, T. Steinel, and M. D. Fayer, Journal of Luminescence 107, 271 (2004).

J. B. Asbury, T. Steinel, and M. D. Fayer, J. Phys. Chem. B 108, 6544 (2004).

C. J. F. Böttcher and P. Bordewijk, Theory of Electric Polarization, 2nd ed.

(Elsevier, Amsterdam, 1978).

G. Sutmann and R. Vallauri, J. Phys.: Condensed Matter 10, 9231 (1998).

G. E. Walrafen, M. S. Hokmabadi, W.-H. Yang, and Y. C. Chu, J. Phys. Chem. 93, 2909 (1989).

Y. C. Chu and G. E. Walrafen, in Hydrogen Bond Networks, edited by M.-C. Bellissent-Funel and J. C. Dore (Kluwer Academic Publishers, Netherlands, 1994), pp. 169.

Quantum corrections, beyond the $\tanh (\beta \hbar v / 2)$ factor in Eq. (1), have not been used to correct the intensity and lineshape of the calculated total dipole spectrum; perhaps this explains the low intensity in the $<50 \mathrm{~cm}^{-1}$ region of the spectrum. M. Sampoli, G. Ruocco, and F. Sette, Phys. Rev. Lett. 79, 1678 (1997). 
J. L. Rousset, E. Duval, and A. Boukenter, J. Chem. Phys. 92 (4), 2150 (1990).

$50 \quad$ R. Chelli, S. Ciabatti, G. Cardini, R. Righini, and P. Procacci, J. Chem. Phys. 111, 4218 (1999).

51 J. Alonso, F. J. Bermejo, M. Garcia-Hernández, J. L. Martínez, W. S. Howells, and A. Criado, J. Chem. Phys. 96 (10), 7696 (1992).

52 J. B. Hasted, S. K. Husain, F. A. M. Frescura, and J. R. Birch, Chem. Phys. Lett. 118, 622 (1985).

53 J. Barthel, K. Buchhuber, R. Buchner, and H. Hetzenauer, Chem. Phys. Lett. 165, 369 (1990).

$54 \quad$ B. Guillot, P. Marteau, and J. Obriot, J. Chem. Phys. 93 (9), 6148 (1990).

55 J.-W. Handgraaf, T. S. van Erp, and E. J. Meijer, Chem. Phys. Lett. 367, 617 (2003).

56 T. S. van Erp and E. J. Meijer, Chem. Phys. Lett. 333, 290 (2001).

57 M. Pagliai, G. Cardini, R. Righini, and V. Schettino, J. Chem. Phys. 119, 6655 (2003).

$58 \quad$ H. R. Zelsmann, J. Mol. Struct. 350, 95 (1995). 